\title{
Amidine Sulfonamides and Benzene Sulfonamides: Synthesis and Their Biological Evaluation
}

\author{
Muhammad Abdul Qadir, ${ }^{1}$ Mahmood Ahmed, ${ }^{1}$ Hina Aslam, \\ Sadia Waseem, ${ }^{1}$ and Muhammad Imtiaz Shafiq ${ }^{2}$ \\ ${ }^{1}$ Institute of Chemistry, University of Punjab, Lahore 54590, Pakistan \\ ${ }^{2}$ Institute of Biochemistry \& Biotechnology, University of the Punjab, Lahore 54590, Pakistan \\ Correspondence should be addressed to Mahmood Ahmed; mahmoodresearchscholar@gmail.com
}

Received 21 May 2015; Accepted 2 July 2015

Academic Editor: Deniz Ekinci

Copyright ( 2015 Muhammad Abdul Qadir et al. This is an open access article distributed under the Creative Commons Attribution License, which permits unrestricted use, distribution, and reproduction in any medium, provided the original work is properly cited.

\begin{abstract}
New amidine and benzene sulfonamide derivatives were developed and structures of the new products were confirmed by elemental and spectral analysis (FT-IR, ESI-MS, ${ }^{1} \mathrm{HNMR}$, and ${ }^{13} \mathrm{CNMR}$ ). In vitro, developed compounds were screened for their antibacterial and antifungal activities against medically important bacterial strains, namely, S. aureus, B. subtilis, and E. coli, and fungi, namely, A. flavus, A. parasiticus, and A. sp. The antibacterial and antifungal activities have been determined by measuring MIC values $(\mu \mathrm{g} / \mathrm{mL})$ and zone of inhibitions $(\mathrm{mm})$. Among the tested compounds, it was found that compounds $\mathbf{3 b} \mathbf{b}, \mathbf{9 a}$, and $\mathbf{9 b}$ have most potent activity against $S$. aureus, A. flavus, and A. parasiticus, respectively, and were found to be more active than sulfamethoxazole and itraconazole with MIC values $40 \mu \mathrm{g} / \mathrm{mL}$. In contrast, all the compounds were totally inactive against the $A$. sp. except $10 \mathrm{~b}$ and 15b to show activity to some extent.
\end{abstract}

\section{Introduction}

Sulfonamides are basis of several drug groups, known as sulfa drugs. Any compound that has sulfonamide moiety $\left(\mathrm{SO}_{2} \mathrm{NH}_{2}\right)$ in its structure is referred to as sulfonamide. They comprise substantial class of pharmaceutical drugs, containing various kinds of pharmacological agents having antitumor [1], antibacterial [2], anticarbonic anhydrase [3, 4], diuretic $[5,6]$, hypoglycemic [7], and protease inhibitory activity [8-10] or antithyroid activity [11] among others. Sulfonamides are mostly used to treat the bacterial infectious cells because they do not significantly affect the antigenic properties of the infective organism or the development of specific antibodies [12]. Bacteria have liability to acquire resistance against sulfonamides by changing their cell wall permeability, enhancing essential metabolites production, or increasing production of enzyme [13]. In this way sulfonamides become ineffective to inhibit their production. But their ineffectiveness in drug therapy can be abstained, due to inductive effect of $\mathrm{SO}_{2}$ group. Sulfonamides having first $\mathrm{p} K_{\mathrm{a}}$ value around 10 are less soluble in water; therefore, they may readily crystalize in kidney but with advancement in medical science, and new sulfonamides have been synthesized having lower $\mathrm{p} K_{\mathrm{a}}$ value (5-6) to avoid crystallization in kidney [14]. The compounds having pyridine and amide functional group exhibit various biological activities like antifungal and antibacterial. So these biological activities encourage us to synthesize the sulfonamides containing such important functional groups [15-18]. The motivation behind this research work was to synthesize some novel sulfonamides (Schemes 1-4) having antimicrobial properties. Different amines were chosen and reacted with sulfonyl chlorides. As a result of substitution, different functional groups were added and resulting compounds exhibited antibacterial and antifungal activities.

\section{Experimental}

2.1. Chemistry. Chemicals used in present work were of analytical grade obtained from E-Merck (Germany), Sigma Aldrich (USA), and BDH (UK) without further purification 


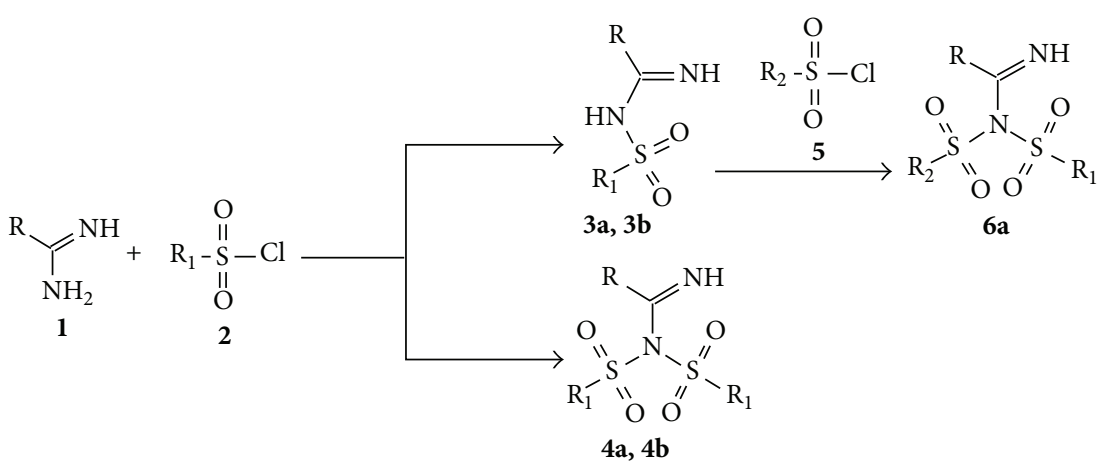

Scheme 1: Amidine sulfonamides.

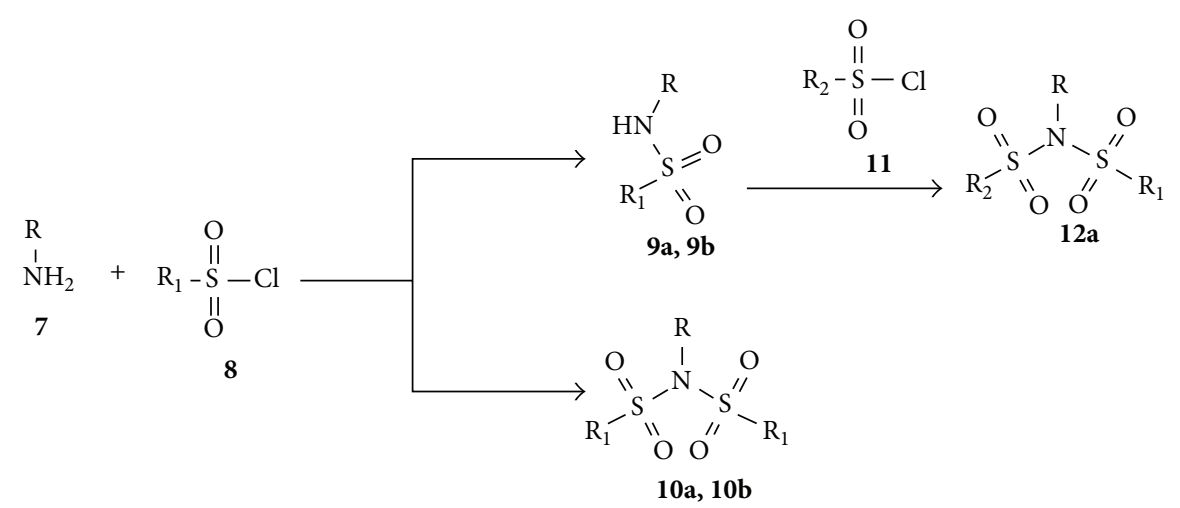

Scheme 2: Benzene sulfonamides (series 1).

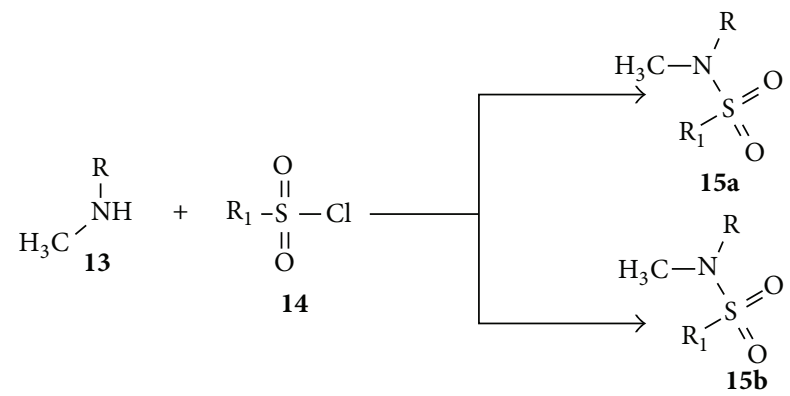

SCHeme 3: Benzene sulfonamides (series 2).

to synthesize desired compounds, and high purity water $(0.01 \mu \mathrm{S} / \mathrm{cm})$ was prepared in our own laboratory using MilliQ purification system (USA). Alpha IR spectrometer (FTIRATR) and NMR spectrometer, Bruker, were used to record the IR and ${ }^{1}$ HNMR (500 MHz) and ${ }^{13} \mathrm{CNMR}(125 \mathrm{MHz})$ spectra, respectively. PG-T80 ${ }^{+} \mathrm{UV}-\mathrm{Vis}$ spectrophotometer (UK) and Flash HT Plus elemental analyzer (Thermo Scientific, UK) were used for $\lambda_{\max }$, and concentration of hydrogen $(\mathrm{H})$, carbon $(\mathrm{C})$, nitrogen $(\mathrm{N})$, and sulfur $(\mathrm{S})$ of synthesized compounds, respectively, while the melting point was measured by Gallenkamp apparatus. JMS-HX-110 spectrometer with electron spray ionization (ESI) interface was used for mass spectra. The ${ }^{1} \mathrm{HNMR}$ and ${ }^{13} \mathrm{CNMR}$ spectra of all the synthesized compounds were measured using $\mathrm{MeOD}$ and concentration of all the compounds was $10-20 \mathrm{mg}$ in $0.8-$ $1.0 \mathrm{~mL}$ of solvent. Purification and progress of the synthesized compounds were confirmed on precoated TLC silica plate (Merck-Germany).

2.2. Antimicrobial Assay. Escherichia coli ATCC 25922, Staphylococcus aureus ATCC 25923, Bacillus subtilis ATCC 6633, Aspergillus flavus ATCC 9643, Aspergillus parasiticus ATCC 15517, and Acremonium sp. ATCC 200667 were collected from Mycology Department, University of Punjab, Lahore, Pakistan, and were maintained in tryptic soy agar (TSA) and potato dextrose agar (PDA) medium, respectively, slants at $5^{\circ} \mathrm{C}$ until use. A series of four 2-fold dilutions (320, 160,80 , and $40 \mu \mathrm{g} / \mathrm{mL}$ ) were made from stock solution of $640 \mu \mathrm{g} / \mathrm{mL}$ in dimethyl sulphoxide (DMSO). All the dilutions were made sterile in an autoclave at $121^{\circ} \mathrm{C}$ for $30 \mathrm{~min}$ with $15 \mathrm{psi}$ pressure after filtration through $0.22 \mu \mathrm{m}$ membrane filter. The minimal inhibitory concentration (MIC) was reported as absence of no observable growth by the lowest concentration of tested compounds after twofold serial dilution. Five individually numbered test tubes with screw caps were sterilized. Tube 1 was filled with $2 \mathrm{~mL}$ of tryptic soy broth culture media including the stock solution of synthesized compounds. $1.0 \mathrm{~mL}$ of this solution was introduced into 2 tubes and diluted with $1.0 \mathrm{~mL}$ culture media and we repeated the procedure up to tube 5 . The tubes were incubated at $25^{\circ} \mathrm{C}$ for $72 \mathrm{hrs}$. Ciprofloxacin and sulfamethoxazole (sulfa drug) were used as reference (positive control to check 


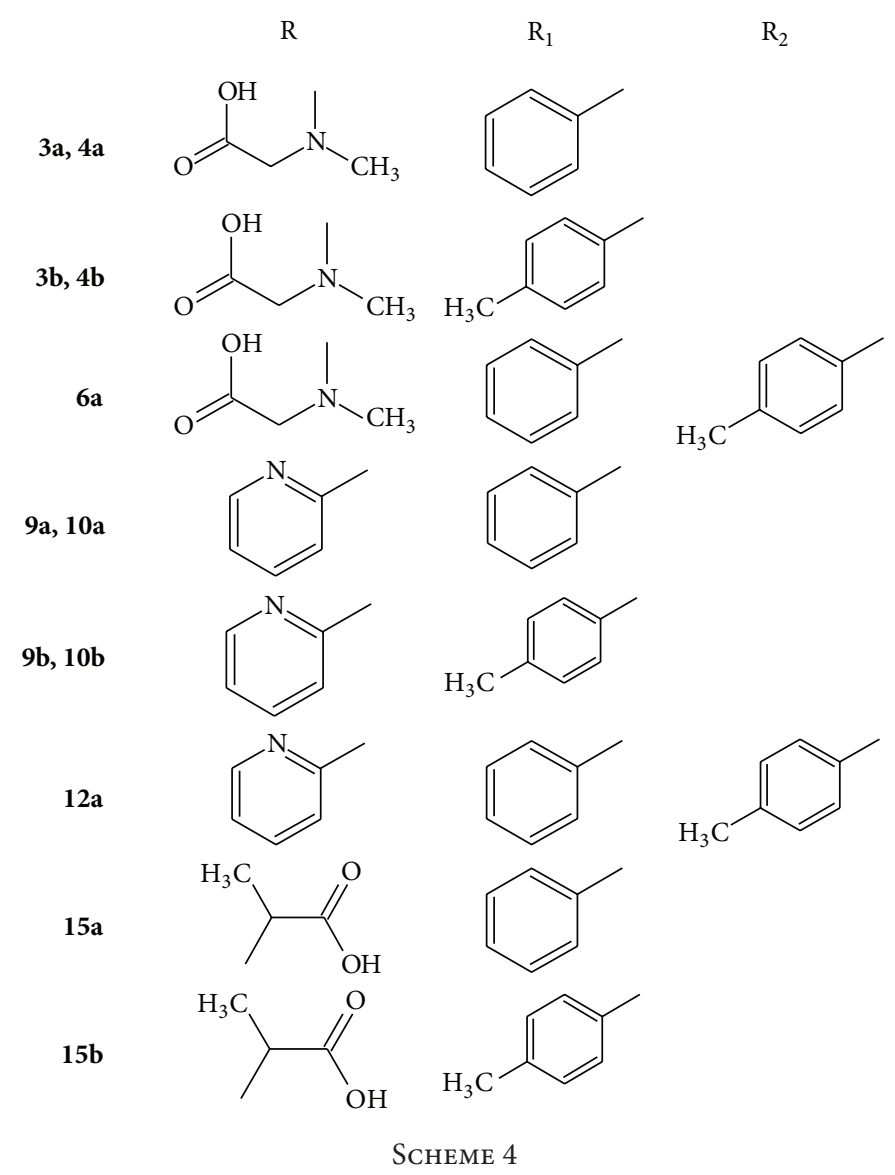

the sensitivity of tested bacterial strains). $1-3 \times 10^{8} \mathrm{cfu} / \mathrm{mL}$ of each of Gram negative E. coli and Gram positive S. aureus and $B$. subtilis was obtained after adjusting the optical density of inoculum at $0.2-0.3$ and $0.3-0.4(620 \mathrm{~nm})$, respectively, while fungal suspension (A. flavus, A. parasiticus, and A. sp.) with cell density of $10^{5} \mathrm{cfu} / \mathrm{mL}$ was studied in present work and the itraconazole was used as reference antifungal agent. All the compounds and reference solutions were applied $(50 \mu \mathrm{L})$ onto a $6 \mathrm{~mm}$ sterile filter paper disc separately and the inoculated plates incubated at $37^{\circ} \mathrm{C}$ for $24 \mathrm{hrs}$. The zones of inhibition ( $\mathrm{mm}$ ) were measured and we evaluated the antibacterial activities.

2.3. General Procedure for Synthesis of Sulfonamides. A simple method in aqueous media under dynamic $\mathrm{pH}$ control is adopted for synthesis of sulfonamides. Filtration after acidification is involved for isolation of products [19-21]. All the amines were weighed accurately and dissolved completely by addition of distilled water by constant stirring using magnetic stirrer. The $\mathrm{pH}$ of the reaction contents was strictly monitored and maintained at $8-10$ at regular intervals during the experimental reaction using $\mathrm{Na}_{2} \mathrm{CO}_{3}$ solution $(1 \mathrm{M})$. Then benzene sulfonyl chloride or p-toluene sulphonyl chloride was accurately weighed and added carefully into the above solution. The reaction was carried in round bottom flask equipped with magnetic stirrer. During stirring sulphonyl chloride initially floats on the surface and the completion

of reaction was examined by the change in $\mathrm{pH}$ value due to formation of $\mathrm{HCl}$ by the consumption of sulphonyl chlorides during the reaction. On completion of the reaction $\mathrm{pH}$ was adjusted at 2-3 using $\mathrm{HCl}$ solution $(2 \mathrm{M})$. The precipitates formed were filtered through Whatman filter paper number 42 , washed several times with distilled water, and recrystallized using methanol and dried using rotary evaporator.

\section{4. $N$-\{Imino[(phenylsulfonyl)amino]methyl $\}-N$-methylglycine} (3a, $\left.\mathrm{C}_{10} \mathrm{H}_{13} \mathrm{~N}_{3} \mathrm{O}_{4} \mathrm{~S}\right)$. Yield: $397.9 \mathrm{mg}$ (64.7\%); m.p.: 172$174^{\circ} \mathrm{C}$; TLC: $R_{f}=0.72\left(\mathrm{H}_{2} \mathrm{O}-\mathrm{BuOH}\right.$-Acetic acid $\left.1: 4: 1\right)$; IR (FTIR): $\bar{v}=3215\left(\mathrm{O}-\mathrm{H}_{\text {carboxylic, stretching }}\right), 3084(\mathrm{C}-$ $\left.\mathrm{H}_{\text {aromatic, stretching }}\right), \quad 1768 \quad\left(\mathrm{C}=\mathrm{O}_{\text {carboxylic, stretching }}\right), \quad 1707$ $\left(\mathrm{C}=\mathrm{N}_{\text {imine, stretching }}\right), \quad 1438 \quad\left(\mathrm{O}-\mathrm{H}_{\text {carboxylic, bending }}\right), \quad 1033$ $\left(\mathrm{S}=\mathrm{O}_{\text {stretching }}\right), 1165 \quad\left(-\mathrm{N}-\mathrm{S}=\mathrm{O}_{\text {stretching }}\right), 1458 \quad(\mathrm{C}=\mathrm{C}-$ $\mathrm{C}_{\text {aromatic, stretching }}$ ), 706 (Ø-S $\mathrm{S}_{\text {stretching }}$ ); UV-Vis (methanol, $c=$ $\left.2 \cdot 10^{-5} \mathrm{~mol} \mathrm{dm}^{-3}\right): \quad \lambda_{\max }(\varepsilon)=208 \quad(25050) \mathrm{nm}$ $\left(\mathrm{mol}^{-1} \mathrm{dm}^{3} \mathrm{~cm}^{-1}\right) ;{ }^{1} \mathrm{HNMR}(500 \mathrm{MHz}, \mathrm{MeOD}) \delta=9.91(\mathrm{br}$, s, $1 \mathrm{H}, \mathrm{NH}), 7.98$ (d, $J=7.48 \mathrm{~Hz}, 1 \mathrm{H}, \mathrm{CH}), 7.68(\mathrm{t}, J=7.59 \mathrm{~Hz}$, $1 \mathrm{H}, \mathrm{CH}), 7.56$ (d, $J=7.63 \mathrm{~Hz}, 1 \mathrm{H}, \mathrm{CH}), 4.71(\mathrm{~d}, J=13.16 \mathrm{~Hz}$, $\left.2 \mathrm{H}, \mathrm{CH}_{2}\right), 3.15\left(\mathrm{~s}, 3 \mathrm{H}, \mathrm{CH}_{3}\right) ;{ }^{13} \mathrm{CNMR}(125 \mathrm{MHz}, \mathrm{MeOD})$ $\delta=168$ (C-16), 155 (C-11), 141 (C-1), 135 (C-4), 129 (C-3), 51 (C-15), 35 (C-14); ESI-MS: $m / z=273.38[\mathrm{M}+2]^{+}, 271.31$ $[\mathrm{M}]^{+}$.

2.5. N-(Imino $\{[(4$ methylphenyl)sulfonyl $]$ amino $\}$ methyl)-Nmethylglycine (3b, $\left.\mathrm{C}_{11} \mathrm{H}_{15} \mathrm{~N}_{3} \mathrm{O}_{4} \mathrm{~S}\right)$. Yield: $779.9 \mathrm{mg}$ (82.7\%); 
m.p.: $185-187^{\circ} \mathrm{C}$; TLC: $R_{f}=0.64\left(\mathrm{H}_{2} \mathrm{O}-\mathrm{BuOH}-\right.$ Acetic acid

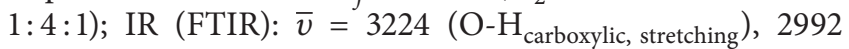
$\left(\mathrm{C}-\mathrm{H}_{\text {aromatic, stretching }}\right), \quad 1767 \quad\left(\mathrm{C}=\mathrm{O}_{\text {carboxylic, stretching }}\right), 1699$ $\left(\mathrm{C}=\mathrm{N}_{\text {imine, stretching }}\right), \quad 1438 \quad\left(\mathrm{O}-\mathrm{H}_{\text {carboxylic, bending }}\right), \quad 1028$ $\left(\mathrm{S}=\mathrm{O}_{\text {stretching }}\right), \quad 1199 \quad\left(-\mathrm{N}-\mathrm{S}=\mathrm{O}_{\text {stretching }}\right), \quad 1498 \quad(\mathrm{C}=\mathrm{C}-$ $\left.\mathrm{C}_{\text {aromatic, stretching }}\right), 701$ ( $\left.c=2 \cdot 10^{-5} \mathrm{~mol} \mathrm{dm}^{-3}\right): \lambda_{\max }(\varepsilon)=222(22500) \mathrm{nm}$ $\left(\mathrm{mol}^{-1} \mathrm{dm}^{3} \mathrm{~cm}^{-1}\right) ;{ }^{1} \mathrm{HNMR}(500 \mathrm{MHz}, \mathrm{MeOD}) \delta=9.96$ (br, s, 1H, NH), $7.71(\mathrm{~s}, 1 \mathrm{H}, \mathrm{CH}), 7.38(\mathrm{~d}, J=8.11 \mathrm{~Hz}, 1 \mathrm{H}$, $\mathrm{CH}), 4.68$ (s, 2H, $\mathrm{CH}_{2}$ ), 3.15 (s, 3H, $\left.\mathrm{CH}_{3}\right), 2.26$ (s, 3H, $\mathrm{CH}_{3}$ ); ${ }^{13} \mathrm{CNMR}$ (125 MHz, MeOD) $\delta=168$ (C-17), 156 (C-12), 143 (C-1), 138 (C-4), 129 (C-2), 52 (C-16), 35 (C-15), 22 (C-7); ESI-MS: $m / z=287.39[\mathrm{M}+2]^{+}, 285.34[\mathrm{M}]^{+}$.

2.6. $N$-[[Bis(phenylsulfonyl)amino](imino)methyl]-N-methylglycine (4a, $\mathrm{C}_{16} \mathrm{H}_{17} \mathrm{~N}_{3} \mathrm{O}_{6} \mathrm{~S}_{2}$ ). Yield: $931.8 \mathrm{mg}$ (81.3\%); m.p.: $210-212^{\circ} \mathrm{C}$; TLC: $R_{f}=0.67\left(\mathrm{H}_{2} \mathrm{O}-\mathrm{BuOH}-A c e t i c\right.$ acid $1: 4: 1)$; IR (FTIR): $\bar{v}=3062\left(\mathrm{O}-\mathrm{H}_{\text {carboxylic, stretching }}\right), 1767$ $\left(\mathrm{C}=\mathrm{O}_{\text {carboxylic, stretching }}\right), 1621\left(\mathrm{C}=\mathrm{N}_{\text {imine, stretching }}\right), 1445(\mathrm{O}-$ $\left.\mathrm{H}_{\text {carboxylic, bending }}\right), 1037\left(\mathrm{~S}=\mathrm{O}_{\text {stretching }}\right), 1163\left(-\mathrm{N}-\mathrm{S}=\mathrm{O}_{\text {stretching }}\right)$, $1445\left(\mathrm{C}=\mathrm{C}-\mathrm{C}_{\text {aromatic, stretching }}\right), 689$ ( $\left.\varnothing-\mathrm{S}_{\text {stretching }}\right)$; UV-Vis (methanol, $c=2 \cdot 10^{-5} \mathrm{~mol} \mathrm{dm}^{-3}$ ): $\lambda_{\text {max }}(\varepsilon)=210(17250) \mathrm{nm}$ $\left(\mathrm{mol}^{-1} \mathrm{dm}^{3} \mathrm{~cm}^{-1}\right) ;{ }^{1} \mathrm{HNMR}$ (500 MHz, MeOD) $\delta=10.86(\mathrm{br}$, $\mathrm{s}, 1 \mathrm{H}, \mathrm{NH}), 8.21(\mathrm{t}, J=7.61 \mathrm{~Hz}, 1 \mathrm{H}, \mathrm{CH}), 8.08(\mathrm{~d}, J=7.61 \mathrm{~Hz}$, $1 \mathrm{H}, \mathrm{CH}), 7.93$ (s, $1 \mathrm{H}, \mathrm{CH}), 4.75\left(\mathrm{~s}, 2 \mathrm{H}, \mathrm{CH}_{2}\right), 3.16$ (s, 3H, $\mathrm{CH}_{3}$ ); ${ }^{13} \mathrm{CNMR}$ (125 MHz, MeOD) $\delta=168$ (C-25), 156 (C-13), 141 (C-1), 132 (C-4), 131 (C-5), 53 (C-24), 37 (C-23); ESI-MS: m/z $=413.59[\mathrm{M}+2]^{+}, 411.48[\mathrm{M}]^{+}$.

2.7. $N$-[\{Bis[(4-methylphenyl)sulfonyl]amino\}(imino)methyl]$\mathrm{N}$-methylglycine $\quad\left(\mathbf{4} \boldsymbol{b}, \quad \mathrm{C}_{18} \mathrm{H}_{21} \mathrm{~N}_{3} \mathrm{O}_{6} \mathrm{~S}_{2}\right)$. Yield: $872.1 \mathrm{mg}$ (82.2\%); m.p.: $195-197^{\circ} \mathrm{C}$; TLC: $R_{f}=0.63\left(\mathrm{H}_{2} \mathrm{O}-\mathrm{BuOH}-\right.$ Acetic acid $1: 4: 1)$; IR (FTIR): $\bar{v}=3265$ (O-H $\mathrm{H}_{\text {carboxylic, stretching }}$ ), $1707\left(\mathrm{C}=\mathrm{O}_{\text {carboxylic, stretching }}\right), 1600\left(\mathrm{~N}-\mathrm{H}_{\text {amine, bending }}\right), 1660$ $\left(\mathrm{C}=\mathrm{N}_{\text {imine, stretching }}\right), \quad 1399 \quad\left(\mathrm{O}-\mathrm{H}_{\text {carboxylic, bending }}\right), \quad 1043$ $\left(\mathrm{S}=\mathrm{O}_{\text {stretching }}\right), \quad 1182 \quad\left(-\mathrm{N}-\mathrm{S}=\mathrm{O}_{\text {stretching }}\right), 1445 \quad(\mathrm{C}=\mathrm{C}-$ $\mathrm{C}_{\text {aromatic, stretching }}$ ), 685 (Ø-S $\left.\mathrm{S}_{\text {stretching }}\right)$; UV-Vis (methanol, $\left.c=2 \cdot 10^{-5} \mathrm{~mol} \mathrm{dm}^{-3}\right): \lambda_{\max }(\varepsilon)=222(19000) \mathrm{nm}$ $\left(\mathrm{mol}^{-1} \mathrm{dm}^{3} \mathrm{~cm}^{-1}\right) ;{ }^{1} \mathrm{HNMR}(500 \mathrm{MHz}, \mathrm{MeOD}) \delta=10.76(\mathrm{br}$, $\mathrm{s}, 1 \mathrm{H}, \mathrm{NH}), 7.81(\mathrm{~d}, J=8.11 \mathrm{~Hz}, 1 \mathrm{H}, \mathrm{CH}), 7.78(\mathrm{~d}, J=8.14 \mathrm{~Hz}$, $1 \mathrm{H}, \mathrm{CH}), 4.73\left(\mathrm{~s}, 2 \mathrm{H}, \mathrm{CH}_{2}\right), 3.15\left(\mathrm{~s}, 3 \mathrm{H}, \mathrm{CH}_{3}\right), 2.46(\mathrm{~s}, 3 \mathrm{H}$, $\left.\mathrm{CH}_{3}\right) ;{ }^{13} \mathrm{CNMR}(500 \mathrm{MHz}, \mathrm{MeOD}) \delta=168(\mathrm{C}-27), 156$ (C-14), 143 (C-1), 139 (C-4), 132 (C-6), 53 (C-26), 37 (C-24), 21 (C-25); ESI-MS: $m / z=441.53[\mathrm{M}+2]^{+}, 439.58[\mathrm{M}]^{+}$.

2.8. $N$-\{Imino[[(4-methylphenyl)sulfonyl](phenylsulfonyl) amino]methyl\}-N-methylglycine $\left(6 a, \quad \mathrm{C}_{17} \mathrm{H}_{19} \mathrm{~N}_{3} \mathrm{O}_{6} \mathrm{~S}_{2}\right)$. Yield: $626.4 \mathrm{mg}$ (70.6\%); m.p.: $182-184^{\circ} \mathrm{C}$; TLC: $R_{f}=0.61$ $\left(\mathrm{H}_{2} \mathrm{O}-\mathrm{BuOH}-\right.$ Acetic acid $\left.1: 4: 1\right) ;$ IR (FTIR): $\bar{v}=3277$ (O- $\left.\mathrm{H}_{\text {carboxylic, stretching }}\right), 1707 \quad\left(\mathrm{C}=\mathrm{O}_{\text {carboxylic, stretching }}\right), \quad 1599$ $\left(\mathrm{N}-\mathrm{H}_{\text {amine, bending }}\right), 1659\left(\mathrm{C}=\mathrm{N}_{\text {imine, stretching }}\right), 1399 \quad(\mathrm{O}-$ $\left.\mathrm{H}_{\text {carboxylic, bending }}\right), 1040\left(\mathrm{~S}=\mathrm{O}_{\text {stretching }}\right), 1185\left(-\mathrm{N}-\mathrm{S}=\mathrm{O}_{\text {stretching }}\right)$, $1444\left(\mathrm{C}=\mathrm{C}-\mathrm{C}_{\text {aromatic, stretching }}\right), 684 \quad\left(\varnothing-\mathrm{S}_{\text {stretching }}\right)$; UV-Vis (methanol, $\left.c=2 \cdot 10^{-5} \mathrm{~mol} \mathrm{dm}^{-3}\right): \lambda_{\max }(\varepsilon)=218(26150) \mathrm{nm}$ $\left(\mathrm{mol}^{-1} \mathrm{dm}^{3} \mathrm{~cm}^{-1}\right) ;{ }^{1} \mathrm{HNMR}(500 \mathrm{MHz}, \mathrm{MeOD}) \delta=10.79(\mathrm{br}$, s, $1 \mathrm{H}, \mathrm{NH}), 8.21$ (d, $J=7.61 \mathrm{~Hz}, 1 \mathrm{H}, \mathrm{CH}), 8.10$ (s, H, CH),
7.91 (s, H, CH), 7.87 (d, J = 8.11 Hz, 1H, CH), 7.73 (s, H, CH), $7.73(\mathrm{~d}, J=8.01 \mathrm{~Hz}, 1 \mathrm{H}, \mathrm{CH}), 4.73\left(\mathrm{~s}, 2 \mathrm{H}, \mathrm{CH}_{2}\right), 3.15$ (s, 3H, $\left.\mathrm{CH}_{3}\right), 2.44\left(\mathrm{~s}, 3 \mathrm{H}, \mathrm{CH}_{3}\right)$; (125 MHz, MeOD) $\delta=168$ (C-26), 156 (C-14), 143 (C-1), 141 (C-17), 132 (C-20), 131 (C-2), 129 (C-3), 53 (C-25), 39 (C-24), 23 (C-7); ESI-MS: $m / z=427.53$ $[\mathrm{M}+2]^{+}, 425.48[\mathrm{M}]^{+}$.

2.9. $\mathrm{N}$-(Pyridin-2-yl)benzene Sulfonamide (9a, $\left.\mathrm{C}_{11} \mathrm{H}_{10} \mathrm{~N}_{2} \mathrm{O}_{2} \mathrm{~S}\right)$. Yield: $869.6 \mathrm{mg}$ (83.1\%); m.p.: $145-147^{\circ} \mathrm{C}$; TLC: $R_{f}=0.61$ $\left(\mathrm{H}_{2} \mathrm{O}-\mathrm{BuOH}\right.$-Acetic acid 1:4:1); IR (FTIR): $\bar{v}=3367(\mathrm{~N}-$ $\left.\mathrm{H}_{\text {amine, stretching }}\right), 1666\left(\mathrm{~N}-\mathrm{H}_{\text {amine, bending }}\right), 1024\left(\mathrm{~S}=\mathrm{O}_{\text {stretching }}\right)$, $1177\left(-\mathrm{N}-\mathrm{S}=\mathrm{O}_{\text {stretching }}\right), 1445\left(\mathrm{C}=\mathrm{C}-\mathrm{C}_{\text {aromatic, stretching }}\right), 687(\varnothing-$ $\mathrm{S}_{\text {stretching }}$ ); UV-Vis (methanol, $c=2 \cdot 10^{-5} \mathrm{~mol} \mathrm{dm}^{-3}$ ): $\lambda_{\max }(\varepsilon)$ $=230(43800) \mathrm{nm}\left(\mathrm{mol}^{-1} \mathrm{dm}^{3} \mathrm{~cm}^{-1}\right) ;{ }^{1} \mathrm{HNMR}(500 \mathrm{MHz}$, $\mathrm{MeOD}) \delta=11.49(\mathrm{br}, \mathrm{s}, 1 \mathrm{H}, \mathrm{NH}), 7.91(\mathrm{~d}, J=5.21 \mathrm{~Hz}, 1 \mathrm{H}, \mathrm{CH})$, $7.81(\mathrm{~d}, J=7.61 \mathrm{~Hz}, 1 \mathrm{H}, \mathrm{CH}), 7.65(\mathrm{t}, J=8.31 \mathrm{~Hz}, 1 \mathrm{H}, \mathrm{CH})$, $7.61(\mathrm{t}, J=7.51 \mathrm{~Hz}, 1 \mathrm{H}, \mathrm{CH}), 7.55(\mathrm{t}, J=7.61 \mathrm{~Hz}, 1 \mathrm{H}, \mathrm{CH})$, $7.25(\mathrm{t}, J=7.21 \mathrm{~Hz}, 1 \mathrm{H}, \mathrm{CH}), 7.15(\mathrm{~d}, J=8.39 \mathrm{~Hz}, 1 \mathrm{H}, \mathrm{CH})$; ${ }^{13} \mathrm{CNMR}$ (125 MHz, MeOD) $\delta=148$ (C-2), 145 (C-6), 140 (C11), 137 (C-4), 132 (C-14), 129 (C-13), 127 (C-12), 115 (C-5), 112 (C-3); ESI-MS: $m / z=236.31[\mathrm{M}+2]^{+}, 234.28[\mathrm{M}]^{+}$.

2.10. 4-Methyl-N-(pyridin-2-yl)benzene Sulfonamide (9b, $\mathrm{C}_{12} \mathrm{H}_{12} \mathrm{~N}_{2} \mathrm{O}_{2} \mathrm{~S}$ ). Yield: $935.6 \mathrm{mg}$ (82.1\%); m.p.: $178-180^{\circ} \mathrm{C}$; TLC: $R_{f}=0.72\left(\mathrm{H}_{2} \mathrm{O}-\mathrm{BuOH}-\right.$ Acetic acid $\left.1: 4: 1\right)$; IR (FTIR): $\bar{v}=3328\left(\mathrm{~N}-\mathrm{H}_{\text {amine, stretching }}\right), 1665\left(\mathrm{~N}-\mathrm{H}_{\text {amine, bending }}\right), 1019$ $\left(\mathrm{S}=\mathrm{O}_{\text {stretching }}\right), \quad 1161 \quad\left(-\mathrm{N}-\mathrm{S}=\mathrm{O}_{\text {stretching }}\right), \quad 1455 \quad(\mathrm{C}=\mathrm{C}-$ $\left.\mathrm{C}_{\text {aromatic, stretching }}\right), 680\left(\varnothing-\mathrm{S}_{\text {stretching }}\right)$; UV-Vis (methanol, $\left.c=2 \cdot 10^{-5} \mathrm{~mol} \mathrm{dm}^{-3}\right): \lambda_{\max }(\varepsilon)=224(15380) \mathrm{nm}$ $\left(\mathrm{mol}^{-1} \mathrm{dm}^{3} \mathrm{~cm}^{-1}\right) ;{ }^{1} \mathrm{HNMR}(500 \mathrm{MHz}, \mathrm{MeOD}) \delta=11.43(\mathrm{br}$, s, $1 \mathrm{H}, \mathrm{NH}), 7.97(\mathrm{~d}, J=8.11 \mathrm{~Hz}, 1 \mathrm{H}, \mathrm{CH}), 7.61(\mathrm{~d}, J=8.31 \mathrm{~Hz}$, $1 \mathrm{H}, \mathrm{CH}), 7.25$ (t, J = 7.21 Hz, 1H, CH), 7.20 (s, 1H, CH), 7.11 (t, $J=8.41 \mathrm{~Hz}, 1 \mathrm{H}, \mathrm{CH}), 7.15(\mathrm{~d}, J=8.39 \mathrm{~Hz}, 1 \mathrm{H}, \mathrm{CH}), 2.48$ (s, 3H, $\left.\mathrm{CH}_{3}\right) ;{ }^{13} \mathrm{CNMR}(125 \mathrm{MHz}, \mathrm{MeOD}) \delta=148$ (C-2), 145 (C-6), 143 (C-14), 137 (C-4), 129 (C-13), 115 (C-5), 111 (C-3), 21 (C-17); ESI-MS: $m / z=250.35[\mathrm{M}+2]^{+}, 248.30[\mathrm{M}]^{+}$.

2.11. N-(Phenylsulfonyl)-N-(pyridin-2-yl)benzene Sulfonamide (10a, $\mathrm{C}_{17} \mathrm{H}_{14} \mathrm{~N}_{2} \mathrm{O}_{4} \mathrm{~S}_{2}$ ). Yield: $909.8 \mathrm{mg}$ (71.7\%); m.p.: 166-168 ${ }^{\circ}$; TLC: $R_{f}=0.62\left(\mathrm{H}_{2} \mathrm{O}-\mathrm{BuOH}\right.$-Acetic acid $1: 4: 1)$; IR (FTIR): $\frac{j}{v}=3348\left(\mathrm{~N}-\mathrm{H}_{\text {amine, stretching }}\right), 1619(\mathrm{~N}-$ $\left.\mathrm{H}_{\text {amine, bending }}\right), 1030\left(\mathrm{~S}=\mathrm{O}_{\text {stretching }}\right), 1186\left(-\mathrm{N}-\mathrm{S}=\mathrm{O}_{\text {stretching }}\right)$, 1445 ( $\left.\mathrm{C}=\mathrm{C}-\mathrm{C}_{\text {aromatic, stretching }}\right), 688 \quad\left(\varnothing-\mathrm{S}_{\text {stretching }}\right)$; UVVis (methanol, $c=2 \cdot 10^{-5} \mathrm{~mol} \mathrm{dm}^{-3}$ ): $\lambda_{\text {max }}(\varepsilon)=215$ (12830) nm $\left(\mathrm{mol}^{-1} \mathrm{dm}^{3} \mathrm{~cm}^{-1}\right)$; ${ }^{1} \mathrm{HNMR}(500 \mathrm{MHz}, \mathrm{MeOD})$ $\delta=8.13(\mathrm{~m}, 1 \mathrm{H}, \mathrm{CH}), 8.11(\mathrm{~m}, 1 \mathrm{H}, \mathrm{CH}), 7.95(\mathrm{~m}, 1 \mathrm{H}, \mathrm{CH}), 7.88$ (m, $1 \mathrm{H}, \mathrm{CH}), 7.81(\mathrm{~m}, 1 \mathrm{H}, \mathrm{CH}), 7.65(\mathrm{qd}, J=8.39 \mathrm{~Hz}, 1.21 \mathrm{~Hz}$, $1 \mathrm{H}, \mathrm{CH}), 7.48(\mathrm{qd}, J=7.29 \mathrm{~Hz}, 5.21 \mathrm{~Hz}, 1 \mathrm{H}, \mathrm{CH}) ;{ }^{13} \mathrm{CNMR}$ (125 MHz, MeOD) $\delta=148$ (C-6), 142 (C-13), 138 (C-2), 131 (C-16), 130 (C-14), 117 (C-5), 113 (C-3); ESI-MS: $m / z=376.45$ $[\mathrm{M}+2]^{+}, 374.44[\mathrm{M}]^{+}$.

2.12. 4-Methyl-N-(phenylsulfonyl)-N-(pyridin-2-yl)benzene Sulfonamide (10b, $\mathrm{C}_{19} \mathrm{H}_{18} \mathrm{~N}_{2} \mathrm{O}_{4} \mathrm{~S}_{2}$ ). Yield: $1140.5 \mathrm{mg}(84.3 \%)$; m.p.: $164-166^{\circ} \mathrm{C}$; TLC: $R_{f}=0.68\left(\mathrm{H}_{2} \mathrm{O}-\mathrm{BuOH}-A c e t i c\right.$ acid $1: 4: 1)$; IR (FTIR): $\bar{v}=3348\left(\mathrm{~N}-\mathrm{H}_{\text {amine, stretching }}\right), 1621(\mathrm{~N}-$ $\left.\mathrm{H}_{\text {amine, bending }}\right), 1027\left(\mathrm{~S}=\mathrm{O}_{\text {stretching }}\right), 1162\left(-\mathrm{N}-\mathrm{S}=\mathrm{O}_{\text {stretching }}\right)$, 
$1445\left(\mathrm{C}=\mathrm{C}-\mathrm{C}_{\text {aromatic, stretching }}\right), 681$ (Ø-S $\left.\mathrm{S}_{\text {stretching }}\right)$; UV-Vis (methanol, $c=2 \cdot 10^{-5} \mathrm{~mol} \mathrm{dm}^{-3}$ ): $\lambda_{\max }(\varepsilon)=222$ (13855) nm $\left(\mathrm{mol}^{-1} \mathrm{dm}^{3} \mathrm{~cm}^{-1}\right) ;{ }^{1} \mathrm{HNMR}$ (500 MHz, MeOD) $\delta=8.19(\mathrm{~s}, \mathrm{H}, \mathrm{CH}), 8.02(\mathrm{~d}, J=8.09 \mathrm{~Hz}, 1 \mathrm{H}, \mathrm{CH}), 7.87(\mathrm{t}$, $J=8.41 \mathrm{~Hz}, 1 \mathrm{H}, \mathrm{CH}), 7.68(\mathrm{~d}, J=8.11 \mathrm{~Hz}, 1 \mathrm{H}, \mathrm{CH}), 7.61(\mathrm{~d}$, $J=8.37 \mathrm{~Hz}, 1 \mathrm{H}, \mathrm{CH}), 7.55(\mathrm{~d}, J=7.27 \mathrm{~Hz}, 1 \mathrm{H}, \mathrm{CH}), 2.46(\mathrm{~s}$, $\left.3 \mathrm{H}, \mathrm{CH}_{3}\right) ;{ }^{13} \mathrm{CNMR}(125 \mathrm{MHz}, \mathrm{MeOD}) \delta=148$ (C-6), $138(\mathrm{C}-$ 2), 132 (C-15), 117 (C-5), 113 (C-3), 22 (C-27); ESI-MS: $m / z=$ $404.55[\mathrm{M}+2]^{+}, 402.51[\mathrm{M}]^{+}$.

2.13. 4-Methyl-N-(phenylsulfonyl)-N-(pyridin-2-yl)benzene Sulfonamide (12a, $\mathrm{C}_{18} \mathrm{H}_{16} \mathrm{~N}_{2} \mathrm{O}_{4} \mathrm{~S}_{2}$ ). Yield: $529.5 \mathrm{mg}$ (65.1\%); m.p.: $188-190^{\circ} \mathrm{C}$; TLC: $R_{f}=0.76\left(\mathrm{H}_{2} \mathrm{O}-\mathrm{BuOH}-A c e t i c\right.$ acid $1: 4: 1)$; IR (FTIR): $\bar{v}=3280\left(\mathrm{~N}-\mathrm{H}_{\text {amine, stretching }}\right), 1620(\mathrm{~N}-$ $\left.\mathrm{H}_{\text {amine, bending }}\right), 1021\left(\mathrm{~S}=\mathrm{O}_{\text {stretching }}\right), 1158\left(-\mathrm{N}-\mathrm{S}=\mathrm{O}_{\text {stretching }}\right)$, $1445\left(\mathrm{C}=\mathrm{C}-\mathrm{C}_{\text {aromatic, stretching }}\right), 682 \quad\left(\varnothing-\mathrm{S}_{\text {stretching }}\right)$; UV-Vis (methanol, $c=2 \cdot 10^{-5} \mathrm{~mol} \mathrm{dm}^{-3}$ ): $\lambda_{\max }(\varepsilon)=220(42550) \mathrm{nm}$ $\left(\mathrm{mol}^{-1} \mathrm{dm}^{3} \mathrm{~cm}^{-1}\right) ;{ }^{1} \mathrm{HNMR}(500 \mathrm{MHz}, \mathrm{MeOD}) \delta=8.15(\mathrm{~d}$, $J=7.69 \mathrm{~Hz}, 1 \mathrm{H}, \mathrm{CH}), 8.07(\mathrm{~d}, J=8.11 \mathrm{~Hz}, 1 \mathrm{H}, \mathrm{CH}), 7.98(\mathrm{~d}$, $J=7.61 \mathrm{~Hz}, 1 \mathrm{H}, \mathrm{CH}), 7.91(\mathrm{t}, J=8.37 \mathrm{~Hz}, 1 \mathrm{H}, \mathrm{CH}), 7.65(\mathrm{~d}$, $J=8.17 \mathrm{~Hz}, 1 \mathrm{H}, \mathrm{CH}), 7.51(\mathrm{~d}, J=7.17 \mathrm{~Hz}, 1 \mathrm{H}, \mathrm{CH}), 7.45(\mathrm{~d}$, $J=7.17 \mathrm{~Hz}, 1 \mathrm{H}, \mathrm{CH}), 2.44\left(\mathrm{~s}, 3 \mathrm{H}, \mathrm{CH}_{3}\right) ;{ }^{13} \mathrm{CNMR}(125 \mathrm{MHz}$, MeOD) $\delta=147$ (C-6), 138 (C-2), 132 (C-15), 117 (C-5), 112 (C-3), 21 (C-27); ESI-MS: $m / z=390.53[\mathrm{M}+2]^{+}, 388.49$ $[\mathrm{M}]^{+}$.

2.14. N-Methyl-N-(phenylsulfonyl)alanine (15a, $\left.\mathrm{C}_{10} \mathrm{H}_{13} \mathrm{NO}_{4} \mathrm{~S}\right)$. Yield: $786.1 \mathrm{mg}$ (51.7\%); m.p.: $132-134^{\circ} \mathrm{C}$; TLC: $R_{f}=0.72$ $\left(\mathrm{H}_{2} \mathrm{O}-\mathrm{BuOH}\right.$-Acetic acid 1:4:1); IR (FTIR): $\bar{v}=3215(\mathrm{O}-$ $\left.\mathrm{H}_{\text {carboxylic, stretching }}\right), 1743\left(\mathrm{C}=\mathrm{O}_{\text {carboxylic, stretching }}\right), 1444$ (O$\left.\mathrm{H}_{\text {carboxylic, bending }}\right), 1041\left(\mathrm{~S}=\mathrm{O}_{\text {stretching }}\right), 1183\left(-\mathrm{N}-\mathrm{S}=\mathrm{O}_{\text {stretching }}\right)$, $1444\left(\mathrm{C}=\mathrm{C}-\mathrm{C}_{\text {aromatic, stretching }}\right), 688 \quad\left(\varnothing-\mathrm{S}_{\text {stretching }}\right) ; \mathrm{UV}-\mathrm{Vis}$ (methanol, $\left.c=2 \cdot 10^{-5} \mathrm{~mol} \mathrm{dm}^{-3}\right): \lambda_{\max }(\varepsilon)=215(13070) \mathrm{nm}$ $\left(\mathrm{mol}^{-1} \mathrm{dm}^{3} \mathrm{~cm}^{-1}\right)$; ${ }^{1} \mathrm{HNMR}(500 \mathrm{MHz}, \mathrm{MeOD}) \delta=7.73(\mathrm{~d}$, $J=7.69 \mathrm{~Hz}, 1 \mathrm{H}, \mathrm{CH}), 7.53(\mathrm{~d}, J=7.51 \mathrm{~Hz}, 1 \mathrm{H}, \mathrm{CH}), 7.36(\mathrm{t}$, $J=7.61 \mathrm{~Hz}, 1 \mathrm{H}, \mathrm{CH}), 4.51(\mathrm{q}, J=7.17 \mathrm{~Hz}, 1 \mathrm{H}, \mathrm{CH}), 3.75(\mathrm{br}$, s, $1 \mathrm{H}, \mathrm{OH}), 2.71\left(\mathrm{~s}, 3 \mathrm{H}, \mathrm{CH}_{3}\right), 1.11\left(\mathrm{~d}, J=7.17 \mathrm{~Hz}, 3 \mathrm{H}, \mathrm{CH}_{3}\right)$; ${ }^{13} \mathrm{CNMR}(125 \mathrm{MHz}, \mathrm{MeOD}) \delta=178$ (C-14), $138(\mathrm{C}-1), 131(\mathrm{C}-$ 4), 62 (C-12), 29 (C-11), 15 (C-13); ESI-MS: $m / z=245.38[\mathrm{M}+$ $2]^{+}, 243.35[\mathrm{M}]^{+}$.

2.15. N-Methyl-N-[(4-methylphenyl)sulfonyl]alanine (15b, $\left.\mathrm{C}_{11} \mathrm{H}_{15} \mathrm{NO}_{4} \mathrm{~S}\right)$. Yield: $910.9 \mathrm{mg}$ (63.6\%); m.p.: $140-142^{\circ} \mathrm{C}$; TLC: $R_{f}=0.70\left(\mathrm{H}_{2} \mathrm{O}-\mathrm{BuOH}-\right.$ Acetic acid $\left.1: 4: 1\right)$; IR (FTIR): $\bar{v}=3268\left(\mathrm{O}-\mathrm{H}_{\text {carboxylic, stretching }}\right), 1710\left(\mathrm{C}=\mathrm{O}_{\text {carboxylic, stretching }}\right)$, $1449\left(\mathrm{O}-\mathrm{H}_{\text {carboxylic, bending }}\right), 1054 \quad\left(\mathrm{~S}=\mathrm{O}_{\text {stretching }}\right), 1146$ $\left(-\mathrm{N}-\mathrm{S}=\mathrm{O}_{\text {stretching }}\right), \quad 1422 \quad\left(\mathrm{C}=\mathrm{C}-\mathrm{C}_{\text {aromatic, stretching }}\right), \quad 677$ $\left(\varnothing-\mathrm{S}_{\text {stretching }}\right.$ ); UV-Vis (methanol, $c=2 \cdot 10^{-5} \mathrm{~mol} \mathrm{dm}^{-3}$ ): $\lambda_{\text {max }}(\varepsilon)=220(17250) \mathrm{nm}\left(\mathrm{mol}^{-1} \mathrm{dm}^{3} \mathrm{~cm}^{-1}\right) ;{ }^{1} \mathrm{HNMR}$ (500 MHz, MeOD) $\delta=7.69(\mathrm{~d}, J=8.09 \mathrm{~Hz}, 1 \mathrm{H}, \mathrm{CH}), 7.32$ (d, $J=7.91 \mathrm{~Hz}, 1 \mathrm{H}, \mathrm{CH}), 4.48(\mathrm{t}, J=7.17 \mathrm{~Hz}, 1 \mathrm{H}, \mathrm{CH}), 3.75$ (br, s, 1H, OH), 2.69 (s, 3H, $\mathrm{CH}_{3}$ ), 2.39 (s, 3H, $\mathrm{CH}_{3}$ ), 1.08 (d, $\left.J=7.17 \mathrm{~Hz}, 3 \mathrm{H}, \mathrm{CH}_{3}\right) ;{ }^{13} \mathrm{CNMR}(125 \mathrm{MHz}, \mathrm{MeOD}) \delta=178$ (C-14), 138 (C-1), 133 (C-4), 129 (C-5), 61 (C-12), 29 (C-11), 15 (C-13); ESI-MS: $m / z=259.38[\mathrm{M}+2]^{+}, 257.35[\mathrm{M}]^{+}$.

\section{Results and Discussion}

A total of twelve novel sulfonamides were synthesized in aqueous basic media by simple reaction of creatine (amidine derivatives), amino pyridine (benzene sulfonamide series 1), and methyl alanine (benzene sulfonamide series 2) with benzene sulfonyl chloride and para toluene sulphonyl chloride, respectively, with continuous stirring and details of reaction conditions are explained in Experimental section and synthetic pathways of sulfonamides are explained in Schemes 1, 2, 3, and 4. Compounds 3a, 3b, 9a, 9b, 15a, and $\mathbf{1 5 b}$ were synthesized in equimolar concentration of creatine, amino pyridine, and methyl alanine with benzene sulphonyl chloride and para toluene sulphonyl chloride, respectively, while 4a, 4b, 9a, and 9b were obtained by bimolar concentration of respective sulphonyl chlorides, respectively. Compounds $\mathbf{6 a}$ and $\mathbf{1 2 a}$ were synthesized by the reaction of $\mathbf{3 b}$ and $\mathbf{9 b}$ with benzene sulphonyl chloride and para toluene sulphonyl chloride in equimolar concentration, respectively. All the compounds except compound 15a (57.5\%) were obtained in good yield. Elemental analysis was performed for the conformation of all the compounds and measurement of absorption maximum $\left(\lambda_{\max }\right)$ provided the justification. The analytical data of synthesized sulfonamides are presented in Table 1. The synthesized compounds were characterized by FT-IR, and the characteristics band at $1621-1707 \mathrm{~cm}^{-1}$ for imine stretching of amidine sulfonamides, $3280-3367 \mathrm{~cm}^{-1}$ of $\mathrm{N}-\mathrm{H}$ amide stretching for benzene sulfonamides (series 1), 3250$3268 \mathrm{~cm}^{-1}$ for $\mathrm{O}-\mathrm{H}$ stretching (benzene sulfonamide series 2 ), and $1082-1199 \mathrm{~cm}^{-1}$ for $(-\mathrm{N}-\mathrm{S}=\mathrm{O})$ and $1019-1054 \mathrm{~cm}^{-1}(\mathrm{~S}=\mathrm{O})$ for all compounds reveals the formation of sulfonamides. [M $+2]^{+}$peaks obtained by ESI-MS represented the isolation of sulfonyl group in all synthesized compounds. The structures of all the compounds were also confirmed by ${ }^{1} \mathrm{HNMR}$ and ${ }^{13}$ CNMR by dissolving in MeOD. ${ }^{1} \mathrm{HNMR}$ spectra of compounds $\mathbf{3} \mathbf{a}$ and $\mathbf{3 b}$ showed a broad signal at $\delta$ 9.91, 9.96 while signal at $\delta$ 10.76-10.86 for $\mathbf{4 a}, \mathbf{4 b}$, and $\mathbf{9 a}$ and $11.43,11.49 \mathrm{ppm}$ for $\mathbf{9 a}$ and $\mathbf{9 b}$ corresponds to $\mathrm{NH}$ group of sulfonamide. A broad singlet at $\delta 3.75 \mathrm{ppm}$ due to $-\mathrm{OH}$ group was also obtained for compounds $\mathbf{1 5 a}$ and $\mathbf{1 5 b}$. The characteristics C-SO-NH signals at $\delta 155-156$ ppm and $\delta 138-$ $140 \mathrm{ppm}$ for amidine and benzene sulfonamides, respectively, were showed by ${ }^{13} \mathrm{CNMR}$ which identified the structures correctly.

Synthesized compounds were also screened for their antibacterial against Gram negative bacteria E. coli and Gram positive $S$. aureus and $B$. subtilis by following the guidelines of CLSI $[22,23]$ using ciprofloxacin and sulfamethoxazole as reference antibacterial agents. Antifungal activity was also evaluated for synthesized compounds against three strains, namely, A. flavus, A. parasiticus, and A. sp, using itraconazole as reference antifungal agent. Among the bacterial strains, compound $\mathbf{3 b}$ has excellent antibacterial activities having MIC $40 \mu \mathrm{g} / \mathrm{mL}$ against $S$. aureus with zone of inhibition comparable with control drug (ciprofloxacin). The $\mathrm{p} K_{\mathrm{a}}$ of compound $\mathbf{3 b}$ is 10.8 which has electron withdrawing inductive effect (mild acidic) for which supports its cell permeability against the particular bacterial strain to bind 
TABLE 1: Physiochemical and analytical data of sulfonamides.

\begin{tabular}{|c|c|c|c|c|c|c|}
\hline \multirow{2}{*}{ Compounds } & \multirow{2}{*}{ Molecular weight } & \multicolumn{5}{|c|}{ Elemental analysis (found/cal.) \% } \\
\hline & & $\mathrm{C}$ & $\mathrm{H}$ & $\mathrm{N}$ & S & ${ }^{\mathrm{a}} \mathrm{p} K_{\mathrm{a}}$ \\
\hline $3 \mathbf{a}$ & 271.29 & $44.33 / 44.27$ & $4.85 / 4.83$ & $15.42 / 15.49$ & $11.83 / 11.82$ & 10.8 \\
\hline $3 b$ & 285.32 & $46.35 / 46.30$ & $5.39 / 5.31$ & $14.68 / 14.73$ & $11.29 / 11.24$ & 10.8 \\
\hline $4 a$ & 411.45 & $46.77 / 46.71$ & $4.15 / 4.16$ & $10.26 / 10.21$ & $15.65 / 15.59$ & 4.7 \\
\hline $4 b$ & 439.51 & $49.17 / 49.19$ & $4.89 / 4.82$ & $9.58 / 9.56$ & $14.57 / 14.59$ & 4.7 \\
\hline $6 a$ & 425.48 & $47.96 / 47.99$ & $4.54 / 4.50$ & $7.88 / 7.98$ & $15.12 / 15.07$ & 4.7 \\
\hline $9 a$ & 234.28 & $56.33 / 56.39$ & $4.28 / 4.30$ & $11.88 / 11.96$ & $13.61 / 13.69$ & 8.5 \\
\hline $9 b$ & 248.30 & $58.03 / 58.05$ & $4.81 / 4.87$ & $11.22 / 11.28$ & $12.85 / 12.91$ & 8.5 \\
\hline $10 \mathrm{a}$ & 374.44 & $54.50 / 54.53$ & $3.68 / 3.77$ & $7.51 / 7.48$ & $17.15 / 17.13$ & 5.3 \\
\hline $10 \mathrm{~b}$ & 402.49 & $56.73 / 56.70$ & $4.52 / 4.51$ & $6.88 / 6.96$ & $15.86 / 15.93$ & 5.3 \\
\hline $12 a$ & 388.46 & $55.63 / 55.65$ & $4.11 / 4.15$ & $7.16 / 7.21$ & $16.46 / 16.51$ & 5.3 \\
\hline $15 \mathbf{a}$ & 243.28 & $49.43 / 49.37$ & $5.41 / 5.39$ & $5.73 / 5.76$ & $13.16 / 13.18$ & 5.0 \\
\hline $15 b$ & 257.31 & $51.33 / 51.35$ & $5.81 / 5.88$ & $5.36 / 5.44$ & $12.49 / 12.46$ & 4.8 \\
\hline
\end{tabular}

${ }^{\mathrm{a}} \mathrm{p} K_{\mathrm{a}}$ values from ACD, Inc.

TABLE 2: Zone ${ }^{a}$ of inhibition and MIC $^{\mathrm{b}}$ of sulfonamides against pathogenic bacterial strains.

\begin{tabular}{|c|c|c|c|c|c|c|}
\hline \multirow{5}{*}{ Compounds } & \multicolumn{6}{|c|}{ Name of bacteria } \\
\hline & \multicolumn{4}{|c|}{ Gram $(+)$ bacterial strains } & \multirow{3}{*}{\multicolumn{2}{|c|}{$\begin{array}{c}\text { Gram }(-) \text { bacterial strains } \\
\text { E. coli } \\
\text { (ATCC 25922) }\end{array}$}} \\
\hline & \multirow{2}{*}{\multicolumn{2}{|c|}{$\begin{array}{c}\text { S. aureus } \\
\text { (ATCC 25923) }\end{array}$}} & \multirow{2}{*}{\multicolumn{2}{|c|}{$\begin{array}{c}\text { B. subtilis } \\
\text { (ATCC 6633) }\end{array}$}} & & \\
\hline & & & & & & \\
\hline & Zone of inhibition & MIC & Zone of inhibition & MIC & Zone of inhibition & MIC \\
\hline $3 a$ &,,--- & - &,,--- & - & $9,-,-$ & $>160$ \\
\hline $3 \mathbf{b}$ & $32,29,26$ & 40 & $8,-,-$ & $>160$ &,,--- & - \\
\hline $4 a$ &,,--- & - &,,--- & - &,,--- & - \\
\hline $4 b$ &,,--- & - &,,--- & - & $28,25,22$ & 80 \\
\hline $6 a$ &,,--- & - &,,--- & - & $9,-,-$ & $>160$ \\
\hline $9 a$ &,,--- & - & $9,-,-$ & 160 & $9,-,-$ & $>160$ \\
\hline $9 b$ &,,--- & - & $8,-,-$ & $>160$ &,,--- & - \\
\hline $10 \mathrm{a}$ & $9,-,-$ & $>160$ &,,--- & - & $14,10,-$ & 160 \\
\hline $10 \mathrm{~b}$ & $27,24,22$ & 80 &,,--- & - &,,--- & - \\
\hline $12 a$ & $9,-,-$ & $>160$ & $28,24,21$ & 80 &,,--- & - \\
\hline $15 a$ & $29,26,21$ & 80 &,,--- & - &,,--- & - \\
\hline $15 b$ &,,--- & - & $27,24,22$ & 80 & $28,25,20$ & 80 \\
\hline Ciprofloxacin $^{c}$ & $36,34,31$ & 0.5 & $36,33,31$ & 0.5 & $35,34,31$ & 0.5 \\
\hline Sulfamethoxazole $^{\mathrm{d}}$ &,,--- & - & $16,13,-$ & 160 & $33,28,25$ & 40 \\
\hline
\end{tabular}

${ }^{\mathrm{a}}$ Zone of inhibition was measured in $\mathrm{mm}$ at concentration of 160,80 , and $40 \mu \mathrm{g} \mathrm{mL}^{-1}$.

${ }^{\mathrm{b}} \mathrm{MIC}$ (minimum inhibitory concentrations) were measured in $\mu \mathrm{g} \mathrm{mL}^{-1}$.

${ }^{\mathrm{c}}$ Control drug; ${ }^{\mathrm{d}}$ sulfa drug.

the proteins. As far as SAR is concerned, amide functional group further supports its activity. Compounds $\mathbf{4 b}$ and $\mathbf{1 5 b}$ showed better activity against $E$. coli and compounds 3 a, 4a, 4b, and 6a have no activity against the Gram positive $S$. aureus and $B$. subtilis while $10 \mathbf{b}, 12 \mathbf{a}, 15 \mathbf{a}$, and $15 \mathbf{b}$ have good activity against these bacterial strains which is higher (MIC $80 \mu \mathrm{g} / \mathrm{mL}$ ) than already existing sulfa drug (sulfamethoxazole; MIC $160 \mu \mathrm{g} / \mathrm{mL}$ ). The MIC values and zone of inhibitions are presented in Table 2. The activities of above-mentioned compounds are their high permeability into cell due to their acidic behavior by virtue of their lower $\mathrm{p} K_{\mathrm{a}}$ values (4.7-5.3), while the $\mathrm{p} K_{\mathrm{a}}$ value of sulfamethoxazole is 5.7. The electrostatic interaction of methyl group with protein and pyridine further supports their antibacterial activities [24].

Among the fungal strains, compounds $\mathbf{9 a}$ and $\mathbf{9 b}$ have excellent activity against $A$. parasiticus and $A$. flavus, respectively, while 10a and 15a showed better activity against $A$. parasiticus and $A$. sp. was insensitive to all strains except compound 15b (MIC $160 \mu \mathrm{g} / \mathrm{mL}$ ). The amide bond formations in the above-mentioned synthesized compounds have excellent activities against the fungal strains as far as the SAR is concerned [25-27]. It is concluded that newly synthesized sulfonamides exhibited greater activity than reference 
TABLE 3: Zone $e^{a}$ of inhibition and $M C^{b}$ of sulfonamides against pathogenic fungal strains.

\begin{tabular}{|c|c|c|c|c|c|c|}
\hline \multirow{4}{*}{ Compounds } & \multicolumn{6}{|c|}{ Name of fungi } \\
\hline & \multirow{2}{*}{\multicolumn{2}{|c|}{$\begin{array}{l}\text { A. parasiticus } \\
\text { (ATCC 15517) }\end{array}$}} & \multirow{2}{*}{\multicolumn{2}{|c|}{$\begin{array}{c}\text { A. flavus } \\
\text { (ATCC 9643) }\end{array}$}} & \multirow{2}{*}{\multicolumn{2}{|c|}{$\begin{array}{c}\text { A. sp. } \\
\text { (ATCC 200667) }\end{array}$}} \\
\hline & & & & & & \\
\hline & Zone of inhibition & MIC & Zone of inhibition & MIC & Zone of inhibition & MIC \\
\hline $3 a$ &,,--- & - &,,--- & - &,,--- & - \\
\hline $3 b$ &,,--- & - & $12,-,-$ & 160 &,,--- & - \\
\hline $4 a$ & $9,-,-$ & $>160$ &,,--- & - &,,--- & - \\
\hline $4 b$ &,,--- & - &,,--- & - &,,--- & - \\
\hline $6 a$ &,,--- & - &,,--- & - &,,--- & - \\
\hline $9 a$ & $24,21,18$ & 40 &,,--- & - &,,--- & - \\
\hline $9 b$ & $22,19,13$ & 80 & $22,19,15$ & 40 & $9,-,-$ & $>160$ \\
\hline $10 a$ & $21,18,12$ & 80 &,,--- & - &,,--- & - \\
\hline $10 \mathrm{~b}$ &,,--- & - & $24,20,18$ & 80 & $13,-,-$ & 160 \\
\hline $12 a$ &,,--- & - & $12,-,-$ & 160 &,,--- & - \\
\hline $15 a$ & $24,21,18$ & 80 &,,--- & - &,,--- & - \\
\hline $15 b$ & $11,-,-$ & $>160$ &,,--- & - & $15,12,-$ & 160 \\
\hline Itraconazole $^{c}$ & $18,11,-$ & 160 & $21,19,15$ & 80 & $12,-,-$ & $>160$ \\
\hline
\end{tabular}

${ }^{\mathrm{a}}$ Zone of inhibition was measured in mm at concentration of 160,80 , and $40 \mu \mathrm{g} \mathrm{mL}^{-1}$.

${ }^{\mathrm{b}} \mathrm{MIC}$ (minimum inhibitory concentrations) were measured in $\mu \mathrm{g} \mathrm{mL}^{-1}$.

${ }^{\mathrm{c}}$ Control drug.

itraconazole. The MIC values and zone of inhibitions are reported in Table 3.

\section{Conclusion}

Amidine and benzene sulfonamides derivatives were synthesized and evaluated biologically. Among the synthesized compounds $\mathbf{3 b}$ was proved potent antibacterial agent with MIC $40 \mu \mathrm{g} / \mathrm{mL}$ and zone of inhibition comparable with ciprofloxacin and more effective than sulfamethoxazole. Synthetic compounds $\mathbf{9 a}$ and $\mathbf{9 b}$ showed better inhibition than itraconazole against $A$. parasiticus and A. flavus, respectively, with MIC $40 \mu \mathrm{g} / \mathrm{mL}$.

\section{Conflict of Interests}

The authors declare that there is no conflict of interests regarding the publication of this paper.

\section{References}

[1] T. Owa and T. Nagasu, "Novel sulphonamide derivatives for the treatment of cancer," Expert Opinion on Therapeutic Patents, vol. 10, no. 11, pp. 1725-1740, 2000.

[2] C. T. Supuran, A. Scozzafava, and A. Casini, "Carbonic anhydrase inhibitors," Medicinal Research Reviews, vol. 23, no. 2, pp. 146-189, 2003.

[3] C. T. Supuran and A. Scozzafava, "Applications of carbonic anhydrase inhibitors and activators in therapy," Expert Opinion on Therapeutic Patents, vol. 12, no. 2, pp. 217-242, 2002.

[4] C. T. Supuran and A. Scozzafava, "Carbonic anhydrase inhibitors," Immunology, Endocrine \& Metabolic Agents in Medicinal Chemistry, vol. 1, no. 1, pp. 61-97, 2001.
[5] T. H. Maren, "Relatons between structure and biological activity of sulfonamides," Annual Review of Pharmacology and Toxicology, vol. 16, pp. 309-327, 1976.

[6] C. Supuran, C. Conroy, and T. Maren, "Carbonic anhydrase inhibitors: synthesis and inhibitory properties of 1,3,4-thiadiazole-2,5-bissulfonamide," European Journal of Medicinal Chemistry, vol. 31, no. 11, pp. 843-846, 1996.

[7] A. E. Boyd III, "Sulfonylurea receptors, ion channels, and fruit flies," Diabetes, vol. 37, no. 7, pp. 847-850, 1988.

[8] S. Thaisrivongs, H. I. Skulnick, S. R. Turner et al., "Structurebased design of HIV protease inhibitors: sulfonamide-containing 5,6-dihydro-4-hydroxy-2-pyrones as non-peptidic inhibitors," Journal of Medicinal Chemistry, vol. 39, no. 22, pp. 43494353, 1996.

[9] C. T. Supuran, A. Scozzafava, and B. W. Clare, "Bacterial protease inhibitors," Medicinal Research Reviews, vol. 22, no. 4, pp. 329-372, 2002

[10] A. Scozzafava and C. T. Supuran, "Carbonic anhydrase and matrix metalloproteinase inhibitors: sulfonylated amino acid hydroxamates with MMP inhibitory properties act as efficient inhibitors of CA isozymes I, II, and IV, and N-hydroxysulfonamides inhibit both these zinc enzymes," Journal of Medicinal Chemistry, vol. 43, no. 20, pp. 3677-3687, 2000.

[11] C. W. Thornber, "Isosterism and molecular modification in drug design," Chemical Society Reviews, vol. 8, no. 4, pp. 563-580, 1979.

[12] Z. Zhong, X. Ji, R. Xing et al., "The preparation and antioxidant activity of the sulfanilamide derivatives of chitosan and chitosan sulfates," Bioorganic \& Medicinal Chemistry, vol. 15, no. 11, pp. 3775-3782, 2007.

[13] R. E. Clopton and A. Smith, "Efficacy of oral sulfadimethoxine against two gregarine parasites, Protomagalhaensia granulosae and Gregarina cubensis (Apicomplexa: Eugregarinida), infecting the Death's Head cockroach, Blaberus discoidalis," Journal of Parasitology, vol. 88, no. 4, pp. 786-789, 2002. 
[14] C. R. Noller and A. J. Castro, "The composition of alkylmagnesium chloride solutions in ethyl ether," Journal of the American Chemical Society, vol. 64, no. 10, pp. 2509-2514, 1942.

[15] X. M. Wang, J. Xu, Y. P. Li et al., "Synthesis and anticancer activity evaluation of a series of $[1,2,4]$ triazolo[1,5-a]pyridinylpyridines in vitro and in vivo," European Journal of Medicinal Chemistry, vol. 67, pp. 243-251, 2013.

[16] L. Li, K. Du, Y. Wang et al., "Self-activating nuclease and anticancer activities of copper(II) complexes with aryl-modified 2,6-di(thiazol-2-yl)pyridine," Dalton Transactions, vol. 42, no. 32, pp. 11576-11588, 2013.

[17] M. S. AlSaid, M. G. El-Gazzar, and M. M. Ghorab, "Anticancer activity of novel thiophenes containing a biological active diphenylsulfone, diazepin, piperidine, oxazepine, acryladehyde and sulfonamide moieties," Drug Research, vol. 63, no. 5, pp. 263-269, 2013.

[18] M. S. Al-Dosari, M. M. Ghorab, M. S. Al-Said, and Y. M. Nissan, "Discovering some novel 7-chloroquinolines carrying a biologically active benzenesulfonamide moiety as a new class of anticancer agents," Chemical and Pharmaceutical Bulletin, vol. 61, no. 1, pp. 50-58, 2013.

[19] X. Deng and N. S. Mani, "A facile, environmentally benign sulfonamide synthesis in water," Green Chemistry, vol. 8, no. 9, pp. 835-838, 2006.

[20] M. A. Qadir, M. Ahmed, and M. Iqbal, "Synthesis, characterization, and antibacterial activities of novel sulfonamides derived through condensation of amino group containing drugs, amino acids, and their analogs," BioMed Research International, vol. 2015, Article ID 938486, 7 pages, 2015.

[21] M. A. Qadir, M. Ahmed, and A. Khaleeq, "Synthesis and biological evaluation of amino terminal modified new sulfonamides of contemporary drugs," Latin American Journal of Pharmacy, vol. 34, no. 4, pp. 719-724, 2015.

[22] Approved Standard M2-A-9, Clinical and Laboratory Standard Institute, Wayne, Pa, USA, 2006.

[23] Clinical and Laboratory Standard Institute, Approved Standard M7-A-9, Clinical and Laboratory Standard Institute, Wayne, Pa, USA, 2006.

[24] B. B. Touré, K. Miller-Moslin, N. Yusuff et al., "The role of the acidity of N-heteroaryl sulfonamides as inhibitors of Bcl-2 family protein-protein interactions," Medicinal Chemistry Letters, vol. 4, no. 2, pp. 186-190, 2013.

[25] V. R. Pattabiraman and J. W. Bode, "Rethinking amide bond synthesis," Nature, vol. 480, no. 7378, pp. 471-479, 2011.

[26] J. F. Soule, H. Miyamura, and S. Kobayashi, "Powerful amide synthesis from alcohols and amines under aerobic conditions catalyzed by gold or gold/iron, -nickel or -cobalt nanoparticles," Journal of the American Chemical Society, vol. 133, no. 46, pp. 18550-18553, 2011.

[27] T. L. Graybill, M. J. Ross, B. R. Gauvin et al., "Synthesis and evaluation of azapeptide-derived inhibitors of serine and cysteine proteases," Bioorganic and Medicinal Chemistry Letters, vol. 2, no. 11, pp. 1375-1380, 1992. 

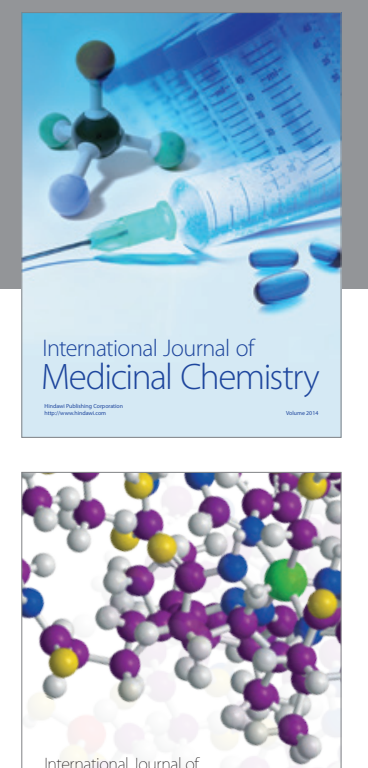

\section{Carbohydrate} Chemistry

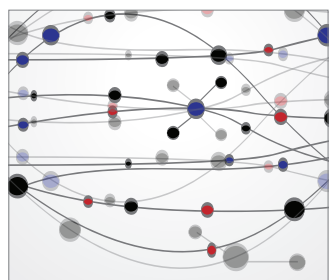

The Scientific World Journal
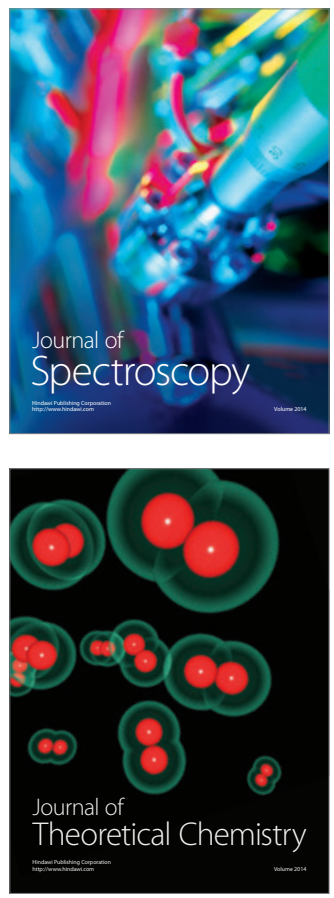
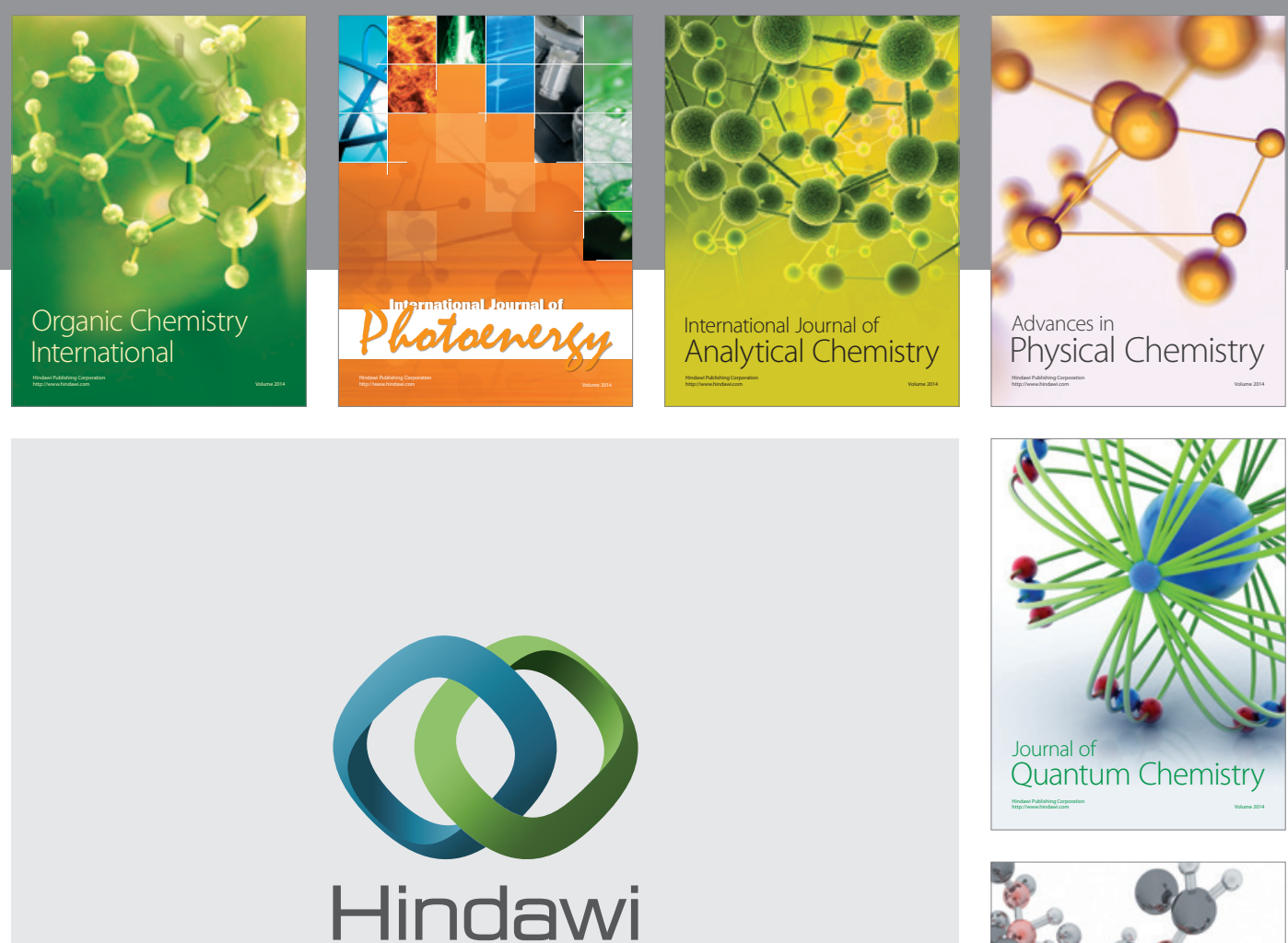

Submit your manuscripts at

http://www.hindawi.com

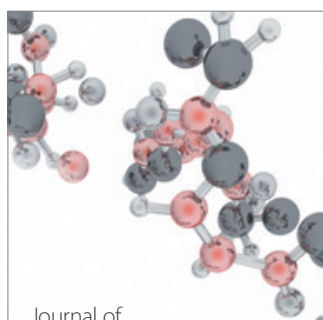

Analytical Methods

in Chemistry

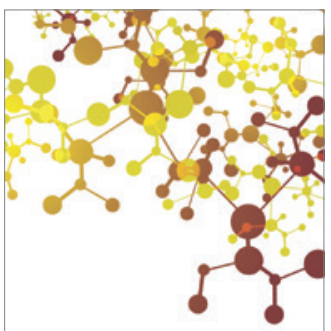

Journal of

Applied Chemistry

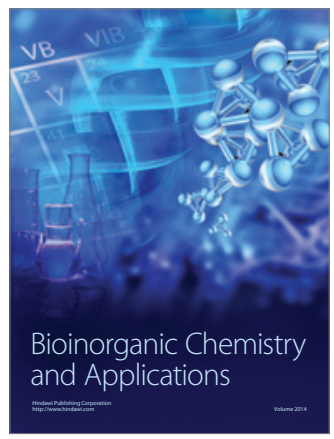

Inorganic Chemistry
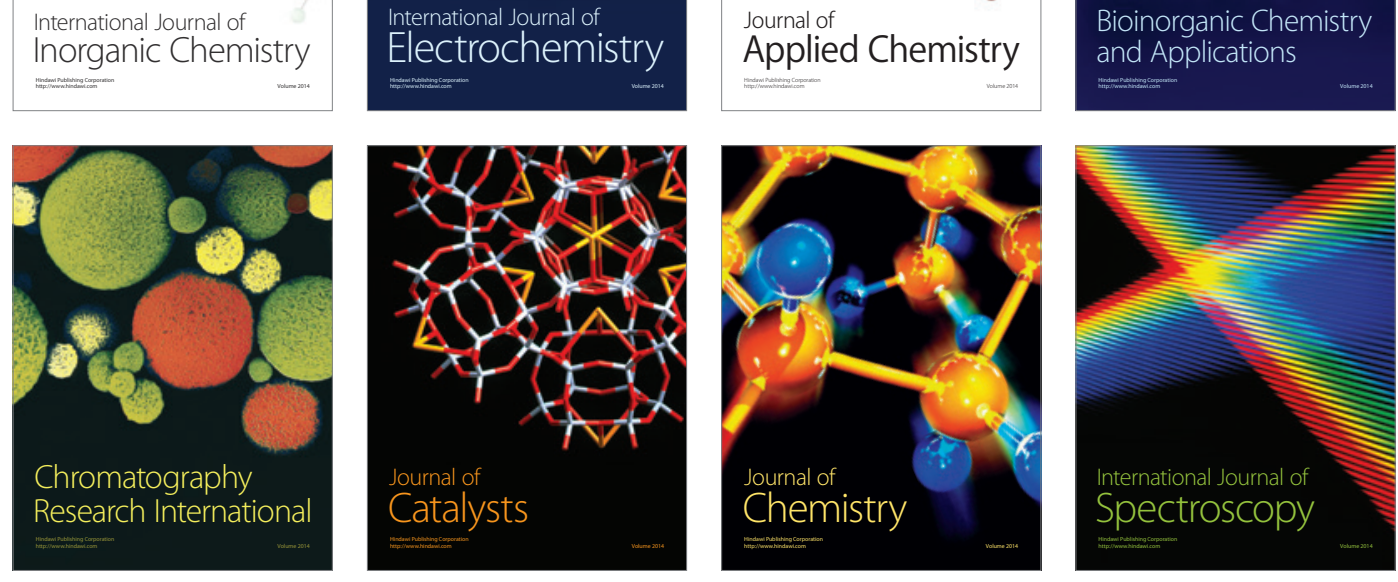